\title{
Consideraciones sobre la dolarización en El Salvador
}

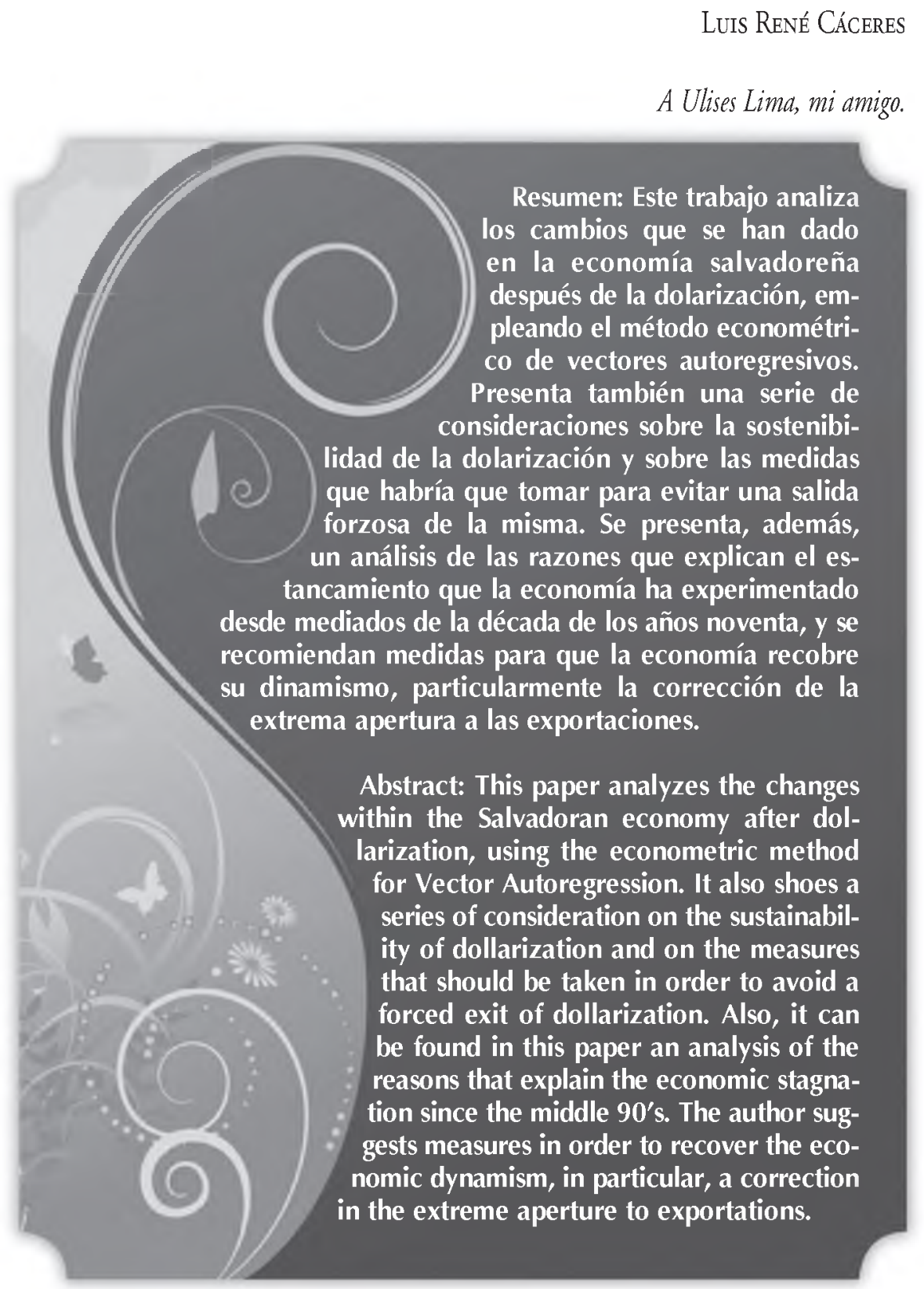




\section{Introducción}

- n enero de 2001, El Salvador adoptó el dólar de los Estados Unidos como moneda de curso legal, iniciando así un proceso de conversión de la moneda local, el colón, a dólares. En pocos meses el colón salió de circulación y el dólar se volvió el medio de intercambio en todas las transacciones. Esta medida causo mucha sorpresa, ya que la dolarización ha sido una medida tomada en respuesta a serios disturbios macroeconómicos, como fue el caso de Ecuador, y cuando Argentina adoptó la caja de conversión, mientras que la economía salvadoreña mostraba la estabilidad que había sido su carácter distintivo en muchas décadas.

Existe una literatura extensa sobre la dolarización financiera, es decir la tenencia de moneda extranjera de parte de los individuos que tratan de proteger sus ahorros de la erosión de su valor a raíz de la inflación' 1 . Sin embargo, no ha habido estudios sobre los cambios experimentados por las variables económicas de una economía que ha sido dolarizada. La intención de este trabajo es explorar las repercusiones de la dolarización en la economía salvadoreña, mediante el análisis de un modelo de vectores regresivos, Var, de demanda de dinero, que incluye las variables en el siguiente orden: índice de actividad económica (Ivae); dinero (M2); índice de precios al consumidor (Ipc); remesas (Remitt); y tasa de interés de corto plazo, la tasa de reportos (Repo) ${ }^{2}$. El modelo Var se calcula con datos mensuales de dos periodos: uno, de enero de 1995 a diciembre de 2000, y el otro, de enero de 2003 a diciembre de 2008. El contraste del comportamiento de las variables incluidas en el Var permite detector los efectos de la dolarización. Todas las variables entran en el Var con sus valores en logaritmos y con rezagos de dos años. En una primera sección se discuten los datos usados en las estimaciones. En secciones subsecuentes se presentan los resultados de estimar el modelo y se consideran las implicaciones de la dolarización. Y el trabajo termina con una serie de consideraciones de política económica de relevancia a la economía salvadoreña.

\section{Datos y vector de cointegración}

Los datos de las variables nacionales se tomaron de la página web del Banco Central de Reserva de El Salvador (BCR). Todas las variables fueron objeto de pruebas de raíces unitarias y en todos los casos se encontró que eran integradas de orden uno. Las pruebas de cointegración indicaron la existencia de un vector de cointegración, y así las ecuaciones autoregresivas fueron estimadas a partir de la ecuación 
de cointegración e incluyen el correspondiente término de error. Los vectores de cointegración para ambos periodos se muestran en la tabla 1.

\section{Tabla 1}

Vectores de cointegración

\begin{tabular}{lcc}
\hline & $\mathbf{1 9 9 5 - 2 0 0 0}$ & $\mathbf{2 0 0 3 - 2 0 0 8}$ \\
\hline $\log$ (Ivae) & 1.0000 & 1.0000 \\
$\log$ (Remittance) & 0.3741 & 0.0464 \\
& $(5.12)$ & $(0.87)$ \\
$\log (\mathrm{M} 2)$ & -1.0692 & -0.0951 \\
& $(8.67)$ & $(1.03)$ \\
$\log ($ Ipc) & 0.6974 & -0.6027 \\
& $(3.53)$ & $(2.62)$ \\
$\log$ (Repo) & -0.1125 & -0.0518 \\
& $(4.83)$ & $(2.76)$ \\
Constante & -1.0882 & -1.4739 \\
\hline
\end{tabular}

Se puede notar que en el segundo periodo las remesas y el dinero, $M 2$, no tienen una relación de largo plazo con el índice de actividad económica. Además, en el segundo periodo, contrario al primero, el índice de precios al con- sumidor ejerce un efecto positivo sobre el Ivae. Estos son los cambios más significativos que se han dado en la relación entre estas variables que, como se verá más adelante, son confirmados por la estimación de los Vars.

\section{Funciones de impulso-respuesta}

Las funciones de impulso respuesta dan información sobre la dirección de una variable en respues- ta a un choque de una desviación estándar en otra variable. Estas funciones se muestran a continuación.
Grúlice 1.

Respuesta de $\log (\operatorname{Repo})$ a choque a $\log (\mathrm{M} 2)$, 1995-2000

Response of LOG(REPO) to Cholesky One S.D. LOG(M2) Innovation

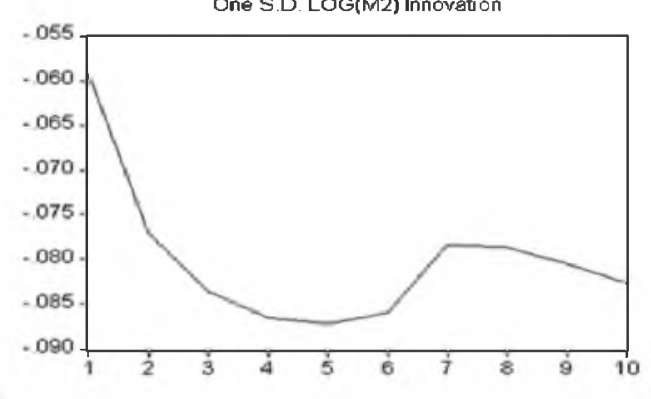

El gráfico 1 presenta la respuesta de $\log (\operatorname{Repo})$ ante un choque de una desviación estándar a $\log (\mathrm{M} 2)$ en el periodo 1995-2000. Se puede ver que la tasa de interés tiene una caída sostenida, lo cual es congruente con el aumento de la liquidez. En contraste, su respuesta en el segundo periodo, que se muestra en el gráfico 2, seña- 
la que la tasa de interés cae solo un poco: la caída es diez veces menor que en el primer periodo. Es decir, la tasa de interés se ha vuelto menos sensible al dinero.

Los gráficos 3 y 4 muestran las respuestas del dinero ante un choque al Ivae. En ambos periodos el dinero aumenta cuando la actividad económica se incrementa, lo que denota la existencia de una demanda de dinero para transacciones. En el primer periodo, la respuesta del dinero es más pronunciada en los primeros meses, pero después del quinto mes las trayectorias de ambos periodos son similares y del mismo orden de magnitud. Esto indica que la demanda de dinero para transacciones existe en ambos periodos.

Se puede ver en el gráfico 5 que la respuesta del Ivae ante un choque al índice de precios al consumidor aumenta en los tres primeros meses y luego cae a valores negativos, lo que se puede interpretar como el resultado de la caída en la demanda agregada como consecuencia de un efecto de riqueza. Sin embargo, en el segundo periodo el Ivae muestra un aumento de gran magnitud que alcanza su valor pico en el
Respuesta de Log(Repo) a choque a Log(M2), 2003-2008

Response of LOG(REPO) to Cholesky One SD LOG(M2) Innovation

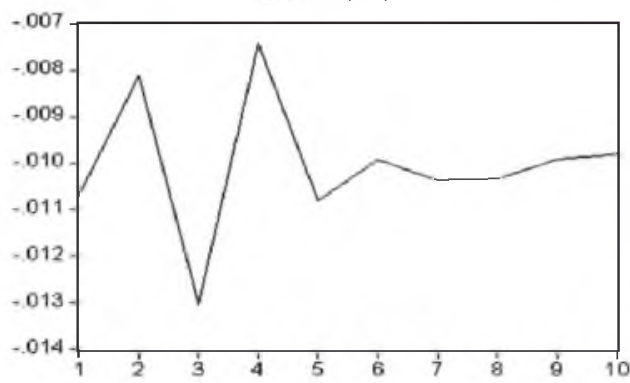

Gráfico 3

Respuesta de $\log (M 2)$ a choque a Log(Ivae), 1995-2000

Response of $L O G(M 2)$ to Cholesky One S.D LOG(IVAE) Innovation

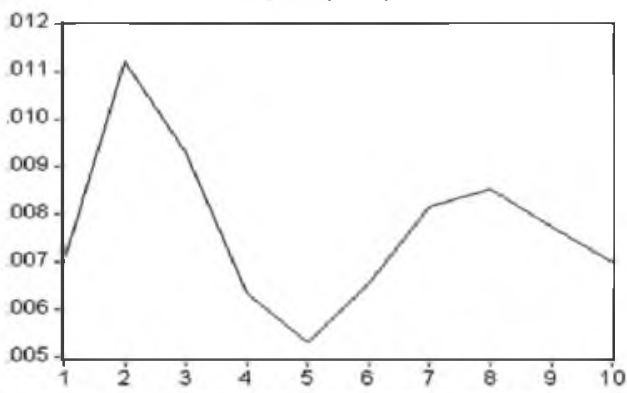

Respuesta de $\log (\mathrm{M} 2)$ ante choque a $\log (\mathrm{Tvae}), 2003-2008$

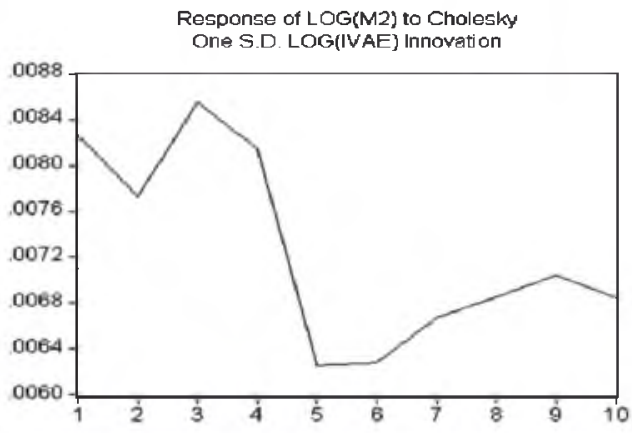


Gráfico 5

Respuesta de Log(Ivae) a choque a Log(Ipc), 1995-2000

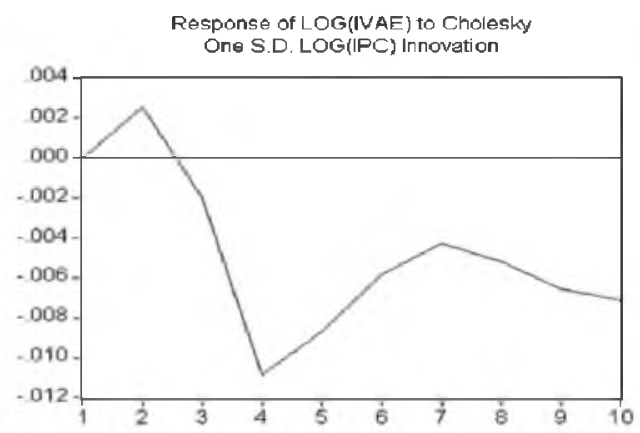

Gráfico 6

Respuesta de $\log (\mathrm{Ivae})$ a choque a $\log (\mathrm{Ipc})$, 2003-2008

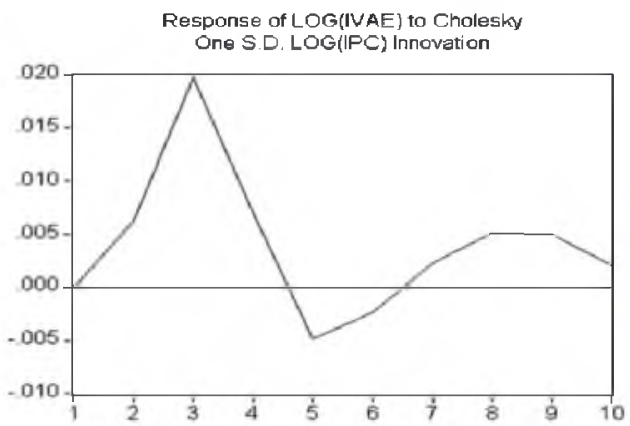

Gráfico 7

Respuesta de Log(Ivae) ante choque a Log(M2), 1995-2000

Response of LOG(IVAE) to Cholesty One S.D. LOG(M2) Innovation

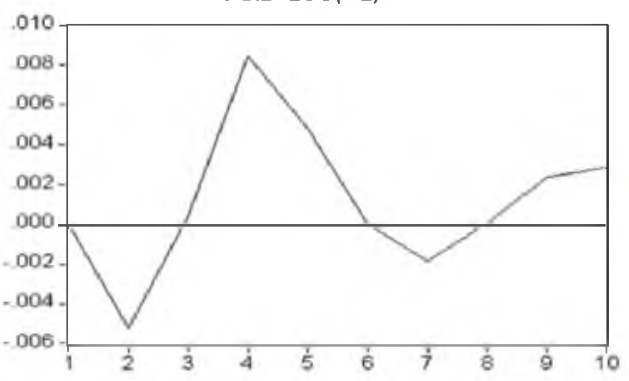

tercer mes, volviéndose negativo en los meses cinco y seis, y positivo desde entonces. Esta respuesta pone en evidencia el comportamiento de la actividad económica como una curva de oferta, ya que responde de manera positiva al aumento de los precios. Asi se puede ver que ha habido cambios sustanciales en la manera que los precios afectan al índice de actividad económica.

El gráfico 7 muestra la respuesta del Ivae a un choque a M2 en el primer periodo. La respuesta inicial es una caída en la actividad económica, que se revierte a valores positivos después de tres meses. Esto indica que en el primer periodo el dinero estimulaba la actividad económica. En contraste, en el segundo periodo el Ivae cae a valores negativos durante todo el periodo en respuesta al aumento del dinero (Gráfico 8).

El gráfico 9 muestra la respuesta del Ivae a un choque a la tasa reporto en el primer periodo. La actividad económica aumenta y alcanza un valor pico al cuarto mes, y disminuye después pero se mantiene positiva. La respuesta en el segundo periodo es negativa en los 


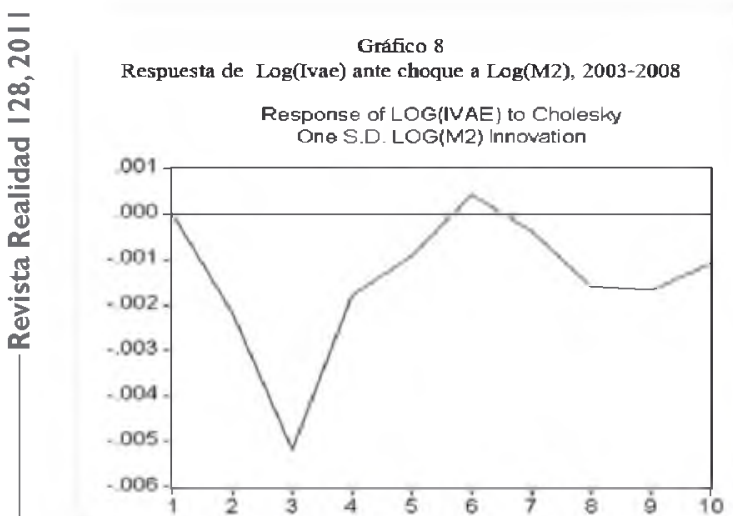

Gráfico 9

Respuesta de $\log (\mathrm{Ivae})$ ante choque a $\log$ (Repo)

Response of LOG(IVAE) to Cholesky One S.D LOG(REPO) Innowation

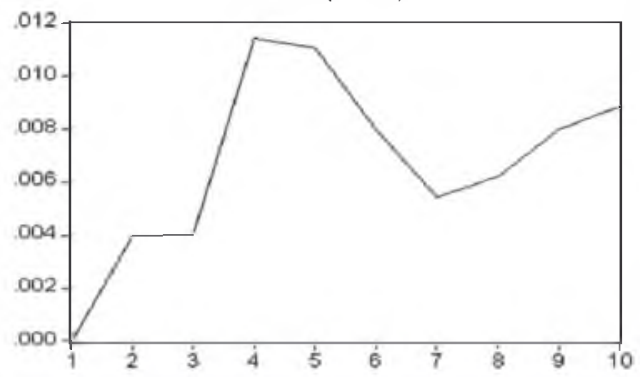

Gráfico 10

Respuesta de Log(Ivae) ante choque a $\log ($ Repo), 2003-2008

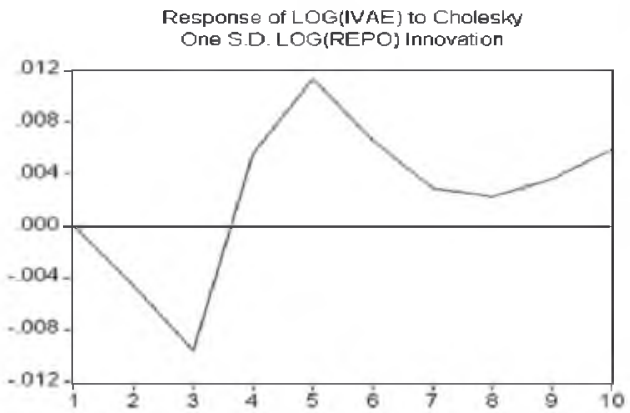

tres primeros meses, y alcanza un valor maximo después de cinco meses y sigue una trayectoria similar al caso previo (Gráfico 10).

\section{Pruebas de causalidad de Granger}

La tabla 2 presenta los resultados de pruebas de causalidad de Granger entre las variables incluidas en el $V r^{3}$. Se puede ver que en el segundo periodo el Ivae ejerce un efecto sobre M2 que es significativo al nivel de $4.82 \%$, más significativo que en el primer periodo. Esto puede ser interpretado como evidencia de la mayor importancia de la demanda de dinero para transacciones.

Se observa también que el Ipc causa en el sentido de Granger al Ivae en el segundo periodo a un nivel de 0.0791 , lo que denota que la actividad económica se comporta como una curva de oferta. Nótese también que en segundo periodo, y contrario al primero, el Ivae causa al Ipc, y la tasa de reportos causa al Ivae. Estos resultados reiteran las fuertes influencias de Ipc y Repo sobre el Ivae, y de este sobre el Ipc. 
Tabla 2. Pruebas de Causalidad de Granger. Hipótesis nula:

La variable 1 no causa la variable 2 .

La variable 2 no causa la variable 1.

\begin{tabular}{lcccc}
\hline \multicolumn{5}{c}{ Períodos } \\
\hline & $\mathbf{1 9 9 5 - 2 0 0 0}$ & \multicolumn{2}{c}{$\mathbf{2 0 0 3 - 2 0 0 8}$} \\
\hline Par de variables & \multicolumn{1}{c}{ Estadístico } & Prob. & Estadístico F & Prob. \\
M2 - Ivae & 0.8617 & 0.5292 & 1.7135 & 0.1159 \\
Ivae - M2 & 2.0238 & 0.0790 & 2.1283 & 0.0483 \\
Ipc - Ivae & 1.4550 & 0.2120 & 1.8927 & 0.0791 \\
Ivae - Ipc & 0.9790 & 0.4489 & 2.5543 & 0.0197 \\
Repo - Ivae & 0.6903 & 0.2120 & 1.8927 & 0.0791 \\
Ivae - Repo & 0.9006 & 0.5017 & 1.3917 & 0.2208 \\
Ipc - M2 & 0.6647 & 0.6784 & 1.4148 & 0.2111 \\
M2 - Ipc & 0.4810 & 0.8194 & 0.7810 & 0.6211 \\
Repo - M2 & 0.6013 & 0.7280 & 2.3443 & 0.0303 \\
M2 - Repo & 0.6063 & 0.7241 & 0.8549 & 0.5595 \\
Repo - Ipc & 0.7950 & 0.5781 & 2.1585 & 0.0452 \\
Ipc - Repo & 1.1443 & 0.3503 & 0.4420 & 0.8904 \\
Libor - Ivae & 0.9080 & 0.4965 & 0.7981 & 0.6069 \\
Ivae - Libor & 1.6938 & 0.1411 & 0.4185 & 0.9050 \\
Libor - Repo & 0.4090 & 0.8699 & 8.5402 & $1.7 \mathrm{E}-07$ \\
Repo - Libor & 2.9715 & 0.0153 & 4.3362 & 0.0004 \\
\hline
\end{tabular}

Se debe notar que no hay causalidad de Granger entre Ipc y M2 en ninguna dirección en los dos periodos. La fuerte causalidad de Granger de la tasa Libor a la tasa de reportos en el segundo periodo es de importancia especial, como es el resultado de que la tasa Libor no afecta al Ivae en ningún periodo, lo que indica que la tasa Libor afecta la actividad económica a través de su efecto sobre el Repo.
Los resultados indican que la cadena de causalidad existente después de la dolarización empieza de las tasas Libor a la Repo, y de esta al Ivae, al Ipc y a la M2, con el Ivae afectando $\mathrm{M} 2$ e IPC, y esta última variable afectando el Ivae (véase gráfico 11). Esto hace ver que el funcionamiento de la economía se origina en el mercado internacional de capitales donde se determina la tasa Libor. 


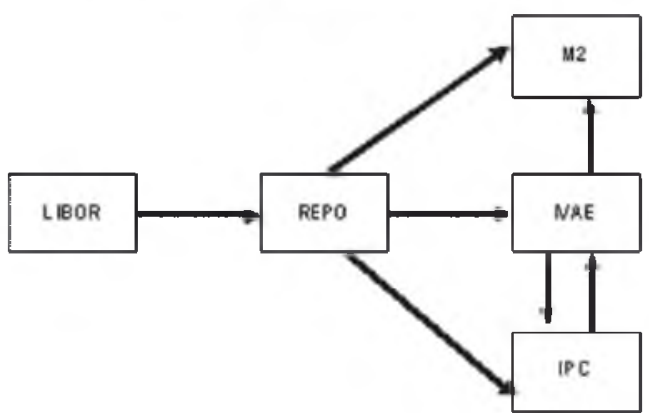

Otras pruebas

Para detectar otros cambios que han ocurrido en el comportamiento de las variables bajo estudio después de la dolarización, se estimaron dos ecuaciones de coin- tegración que expresan la tasa de interés activa (Ractiva) en función de la tasa Libor, con los resultados siguientes:

Tabla 3

\begin{tabular}{lrr}
\hline & \multicolumn{2}{c}{ Período } \\
\cline { 2 - 3 } & $\mathbf{1 9 9 5 - 2 0 0 0}$ & $\mathbf{2 0 0 3 - 2 0 0 8}$ \\
\hline $\log$ (Ractiva) & 1.0000 & 1.0000 \\
$\log$ (Repo) & -0.2726 & -0.3084 \\
& $(1.99)$ & $(7.58)$ \\
Constante & 1.8103 & -1.5940 \\
\hline
\end{tabular}

Se puede ver que el coeficiente de $\log ($ Repo) es más grande y más significativo en el segundo periodo, mientras en el primero este coeficiente apenas era significativo al nivel de cinco por ciento. Esto indica que la tasa Repo ejerce un efecto mucho mas fuerte sobre la tasa activa en el segundo periodo.
La tabla 4 muestra las ecuaciones de cointegracion que expresan el monto de préstamos activos al sector privado (Prest), en función del Ivae y la tasa Libor. Se nota que en ambos periodos el Ivae tiene coeficientes que son significativos y negativos, como se esperaba. En el primer periodo el coeficiente de Li- 
bor no es significativo, mientras que en el segundo es muy significativo y positivo, indicando que cuando esta tasa aumenta la demanda de crédito disminuye.

\section{Tabla 4}

\section{Ecuaciones de cointegración de la carrera de préstamos al sector privado}

\begin{tabular}{lcc}
\hline & \multicolumn{2}{c}{ Período } \\
\cline { 2 - 3 } & $\mathbf{1 9 9 5 - 2 0 0 0}$ & $\mathbf{2 0 0 3 - 2 0 0 8}$ \\
\hline $\log$ (Loans) & 1.0000 & 1.0000 \\
$\log$ (Ivae) & -3.1236 & -2.9242 \\
& $(10.71)$ & $(13.21)$ \\
$\log$ (Libor) & 0.2415 & 0.0739 \\
& $(1.36)$ & $(2.58)$ \\
Constante & 7.1095 & 6.5242 \\
\hline
\end{tabular}

\section{Tipo de cambio efectivo real}

De importancia especial es observar el Índice del Tipo de Cambio Efectivo Real, ITCERES, después de la dolarización. Como se puede ver en el gráfico 12, este índice muestra una trayectoria ascendente en años recientes, lo que denota una tendencia a la depreciación, que se traduce en mayor competitividad de las exportaciones salvadoreñas. La explicación de este comportamiento reside en el hecho de que una parte considerable de las exportaciones salvadoreñas se destinan a los otros países centroamericanos, los cuales han experimentado tasas de inflación más altas que las de El Salvador y de esa manera inducen una depreciación al ITCERES salvadoreño. Este "efecto de barrio" es un resultado no esperado de la dolarización; se puede suponer que mientas El Salvador mantenga tasas de inflación más bajas que las de los otros países de la región, la tendencia de depreciación de la moneda va a continuar. Esto debería verse como un incentivo para aumentar las inversiones en rubros de exportaciones hacia Centroamérica y así capitalizar la competitividad derivada del tipo de cambio efectivo real en la región. 
Una discusión de diversas posiciones sobre las ventajas y desventajas de los regímenes cambiarios se presenta en el Anexo 1, el cual será publicado en la segunda entrega de este artículo.

\section{Tasas activas y pasivas y su diferencial}

Se puede observar en el gráfico 13 la caída drástica de la tasa activa, (Rlending), y de depósito (Rdeposit) en 2001, lo que refleja la eliminación del riesgo cambiario.

La trayectoria del diferencial (spread), entre tasas activa y pasiva en el periodo 1995 y 2008 se muestra en el gráfico 14. Se puede observar que el diferencial disminuye entre 1995 y 1998 y empieza a aumentar hasta el fin de 2000. El diferencial cae hasta 2004 y a partir de entonces no muestra un patrón definido excepto por la tendencia al alza en 2008.

\section{¿Valió la pena dolarizar?}

El resultado más palpable de la dolarización ha sido la caída de las tasas de
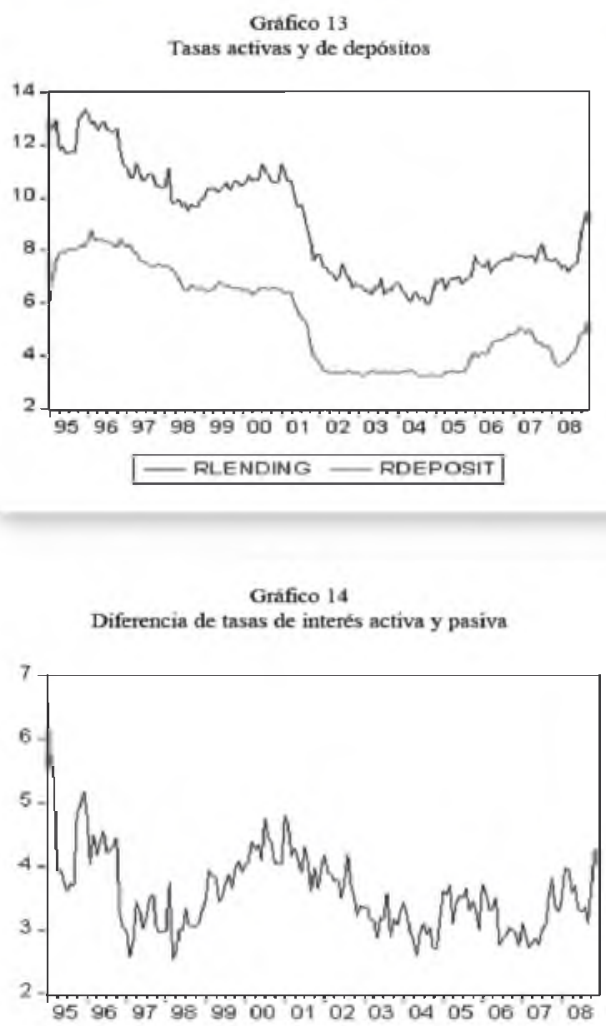

- SPREAD interés, lo que ha contribuido a la expansión del mercado hipotecario en condiciones atractivas. No obstante, habría que considerar que la tasa de depósito ha caído sustancialmente, lo que podría tener repercusiones sobre la movilización del ahorro nacional, dada la evidencia de que la tasa de depósito

estimula el ahorro interno. (Giovannini, 1985). Las tasas de inflación también han bajado después de la dolarización.

Con la dolarización ha mermado la capacidad del banco central de conducir la política monetaria, 
ya que la masa de dinero, así como los precios y el lvae obedecen a la tasa Libor. Esto implica una mayor vulnerabilidad de la economía salvadoreña ante los mercados internacionales de capital, así como mayores dificultades para lograr la estabilización económica y el desarrollo económico.

Lo que también se ha perdido es la capacidad de mejorar la competitividad de las exportaciones mediante la devaluación nominal. Esto puede tener repercusiones adversas, no sólo sobre el dinamismo de las exportaciones, sino también sobre el empleo, dado que varios estudios evidencian que la devaluación nominal conduce a aumentar el empleo (Burgess y Knetter, 1998; Kandil y Mirzaie, 2003; Goldberg y Tracy, 2000), lo cual ha sido corroborado para el caso centroamericano (Cáceres, 2009C). Además, no ha habido cambios positivos en la generación de empleo, o en el crecimiento económico, ni en la inversión, que se pueden atribuir a la dolarización. Lo anterior hace ver que los costos de la dolarización superan sus beneficios. Pero el tema a recalcar es el diseño de políticas para recuperar el dinamismo económico e impulsar la generación de buenos empleos, lo cual no está asociado exclusivamente con la dolarización.

Hay que tener presente que abandonar la dolarización traería costos muy altos. Basta recordar los altos costos que experimentó Argentina cuando salió del esquema de caja de conversión, que, entre otros aspectos, significó la moratoria en el pago de la deuda, la congelación de depósitos en el sistema bancario, y el aumento de la pobreza a cerca de $50 \%$. Argentina ha superado este trauma económico gracias a su extensa oferta exportable, a los altos precios de exportaciones y a la abundancia de recursos humanos calificados ${ }^{4}$. Este no es el caso en El Salvador, cuya producción de bienes transables ha ido disminuyendo en las últimas décadas, y su economía es en efecto una economía de importación.

Sin embargo, abandonar la dolarización bien puede ser un escenario al que forzosamente se podría llegar ante dificultades de pagar la deuda externa y el cierre de fuentes de financiamiento externo. Esto podría asociarse a aumentos de las tasas de interés internacionales, o como resultado de aumentos en la deuda externa como medio para atender el déficit fiscal. Pero reducir el gasto público con miras a reducir el endeudamiento no es una medida apropiada ya que atizaría el deterioro social, lo que acentuaría el estancamiento económico. El escenario de la desdolarización forzada podría ocurrir también como consecuencia de la reducción de las remesas, lo que podría implicar el colapso del consumo que se ha desarrollado alrededor de las mismas, y la consecuente crisis bancaria. 
Lo que procede en la situación de la economía salvadoreña es incrementar sustancialmente los ingresos fiscales y así atender las brechas sociales y ampliar la base de capital humano, de manera que se pueda contar con los recursos humanos necesarios para dotar a la economía de productividad y ampliar la base exportadora.

A continuación se discuten algunas medidas idóneas para potenciar las finanzas públicas y el crecimiento económico.

\section{Variables que inciden en el crecimiento económico}

En este punto debe hacerse una referencia a la vasta literatura en relación al papel determinante que la calidad de las instituciones y del capital humano ejerce sobre la inversión y el crecimiento. ${ }^{5}$ En este respecto, la situación de El Salvador señala que hay mucho que hacer.
La tabla 5 muestra los índices de gobernabilidad del país calculados por el Banco Mundial (Kaufmann, Kraay y Mastruzzi, 2008) y se puede apreciar que son más bajos que los de Panamá y Costa Rica, y superiores a los del resto de los países centroamericanos.

Tabla 5

Índices de gobernabilidad (2007)

\begin{tabular}{lcccc}
\hline \multicolumn{1}{c}{ País } & $\begin{array}{c}\text { Rendición de } \\
\text { cuentas }\end{array}$ & $\begin{array}{c}\text { Efectividad del } \\
\text { gobierno }\end{array}$ & $\begin{array}{c}\text { Estado de } \\
\text { derecho }\end{array}$ & $\begin{array}{c}\text { Control de } \\
\text { corrupción }\end{array}$ \\
\hline El Salvador & 0.07 & -0.23 & -0.68 & 0.02 \\
Costa Rica & 0.88 & 0.39 & 0.44 & 0.39 \\
Panamá & 0.52 & 0.25 & -0.20 & - \\
Chile & 0.98 & 1.22 & 1.17 & 1.35 \\
Guatemala & -0.30 & -0.59 & -1.11 & -0.75 \\
Honduras & -0.23 & -0.57 & -0.86 & -0.69 \\
Nicaragua & -0.10 & -0.91 & -0.84 & -0.78 \\
\hline
\end{tabular}

Existe una extensa literatura que postula que las instituciones son el principal elemento determinante del crecimiento y del dinamismo de las exportaciones ${ }^{6}$. Se ha calculado que si los países latinoamericanos tuviesen niveles de gobernabilidad similares a los de los países de la Unión Europea, sus exportaciones aumentarían en 45\% (Anderson y Marcouiller, 2002). En el caso de una muestra de países latinoamericanos, Cáceres (2008b, 2009a) ha mostrado que sus exportaciones per cápita dependen de sus niveles de capital humano y de gobernabilidad. Así mismo, este autor (2008a, 2009a) ha mostrado 
que las instituciones dan lugar a un efecto multiplicador sobre el PIB de magnitud similar al multiplicador resultante de aumentos del gasto público.
Esta evidencia debería conducir a que el aumento de la calidad de las instituciones y del capital humano ocupara un lugar prominente en las políticas nacionales de desarrollo.

\section{Educación}

Muchos estudios han evidenciado la importancia del capital humano en impulsar el crecimiento económico. En el caso de una muestra de países latinoamericanos, Baffes y Shah (1998) han demostrado que el capital humano ejerce un efecto sobre el crecimiento económico que es mayor que el que resulta de la inversión física y las exportaciones. Además, hay evidencia de que la educación temprana tiene un importante papel en la determinación de los logros educacionales de un individuo ${ }^{7}$, y que nutrición en la edad temprana conduce a mejorar el desempeño de un individuo en el mercado laboral ${ }^{8}$. Estos estudios demuestran de manera contundente que el desarrollo socio-económico depende en gran parte en la atención de las demandas de capital humano desde la edad temprana.

La tabla 6 muestra los niveles de capital humano en los países centroamericanos a principios de los años 2000. Los niveles más altos corresponden a Costa Rica, lo que explica sus éxitos en material de exportaciones, no obstante que tiene salarios más altos, tipo de cambio flexible y regulaciones al sector privado más rigurosas que las de los otros países. Asimismo, los altos niveles de capital humano de Panamá ayudan a explicar su especial dinamismo económico de años recientes. Los datos de El Salvador indican que hay mucho que se debe hacer en materia de educación si se quiere tener una economía dinámica, con equidad y buenos empleos. El hecho es que no hay evidencia de que exista otra variable con más efecto determinante sobre el crecimiento económico que el capital humano; aun mas, la evidencia histórica muestra que el desarrollo económico y social es precedido de cuantiosos avances en educación (Esterlin, 1981). 
Tabla 6 Índices de capital humano*

\begin{tabular}{lcc}
\hline & Años promedio de escolaridad & \\
& Urbano & Rural \\
\hline El Salvador & 8.32 & 3.53 \\
Costa Rica & 9.02 & 6.15 \\
Panamá & 10.84 & 7.04 \\
Chile & 10.83 & 6.77 \\
Guatemala & 6.28 & 3.10 \\
Honduras & 7.41 & 3.64 \\
Nicaragua & 7.28 & 2.91 \\
\hline
\end{tabular}

\section{Exportaciones}

Existe una extensa literatura sobre los efectos que las exportaciones ejercen sobre el crecimiento económico ${ }^{9}$. En el caso de El Salvador, las exportaciones tienen una importancia especial, dado su papel en la creación de liquidez a través de la balanza de pagos. Una corriente de la literatura reciente ha argumentado que la inversión en bienes transables, ya sea para la exportación o para el consumo doméstico, es una característica que distingue a los países que han alcanzado altas tasas de crecimiento económico (Rodrik, 2009a). Este autor aboga el caso de la adopción de políticas industriales que promuevan la inversión en tal sector, recalcando la importancia de subsidios, tales como préstamos bancarios con bajas tasas de interés, entrenamiento de trabajadores, depreciación de la moneda, entre otros.
Se debe apuntar que en El Salvador existe una relación muy clara entre el logaritmo de las exportaciones, $\log (E X)$, en millones de dólares constantes de 1990, y el de la tasa de inversión privada, $\log ($ IPR), con rezagos de dos años, de tal manera que, como se puede observar en el gráfico 15, la inversión privada como porcentaje del PIB en un año dado da lugar a un aumento de las exportaciones dos años después ${ }^{10}$.

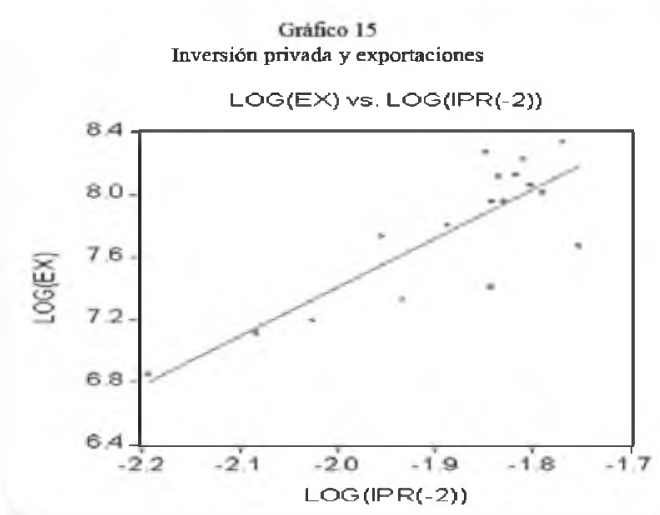


Además, se debe apuntar que en El Salvador el logaritmo de la tasa de inversión privada responde al aumento del logaritmo de la tasa de inversión pública, $\log (\mathrm{IGR})$, con un rezago de dos años, como se aprecia en el gráfico 16.

Estas relaciones indican que el aumento de la inversión pública en un año dado resultará en un aumento de la tasa de inversión privada dos años después, y consecuentemente, en un aumento de las exportaciones cuatro años después.

Se puede deducir que el crecimiento de las exportaciones, que tradicionalmente se ha visto como el medio para dinamizar el crecimiento económico, depende en parte, del dinamismo de la inversión pública, lo que subraya la importancia de contar con suficientes recursos fiscales para incrementarla. Es decir, aumentar la recaudación fiscal y destinarla a la inversión pública resultaría en aumentos de las exportaciones en el mediano plazo. Este es un proceso que se nota en otros países latinoamericanos ${ }^{11}$.

El proceso descrito conduce a aumentar la tasa de crecimiento económico, dada la asociación entre las exportaciones y el PIB, como se observa en el gráfico.
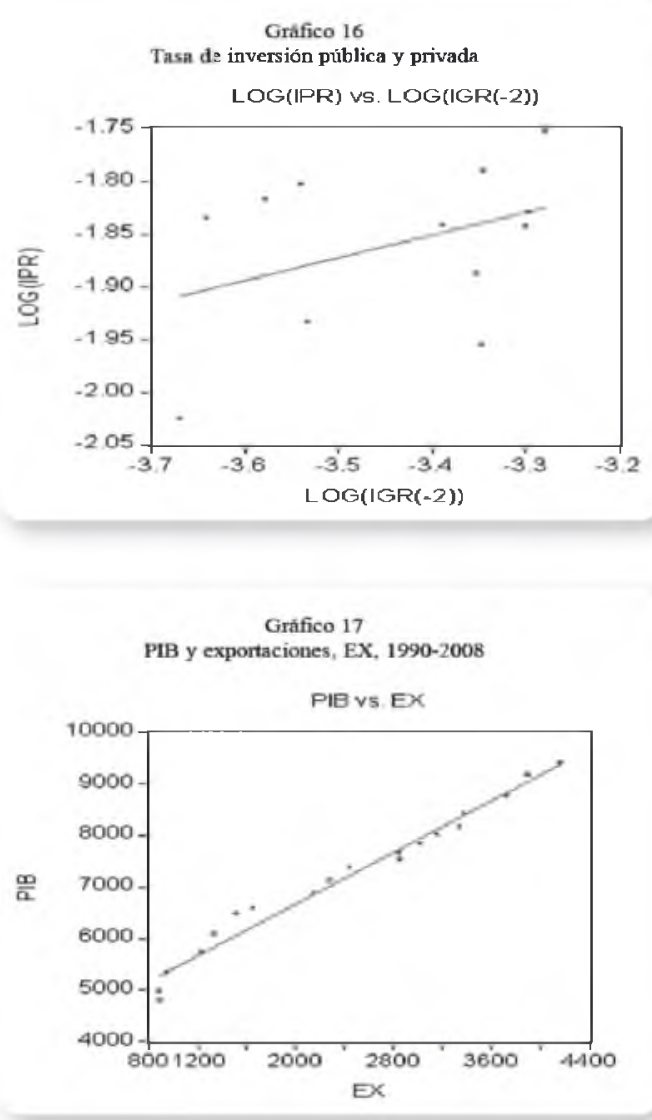

Se debe recalcar que aumentar la recaudación fiscal y destinarla a la inversión pública es una buena práctica de negocios, dado que conduce a incrementar la inversión privada y las exportaciones aéreas cuyo dinamismo ha sido elusivo en las últimas décadas. $Y$ también conduciría a aumentar las ganancias de las empresas del sector privado, lo que hace ver que su carga impositiva pagada sería mayor que la carga neta, una vez que de esta se descuentan los beneficios de la 
inversión pública adicional generados gracias a los impuestos. Existe evidencia de que las inversiones en infraestructura y en educación se pagan a sí mismas, dado que sus beneficios más que compensan los impuestos pagados ${ }^{12}$. De hecho, rigurosas evaluaciones de programas de educación preescolar han encontrado que bastan sus beneficios en términos de la reducción del crimen para que estos programas se paguen asi mismos (Donahue $y$ Siegelman, 1998)

Además, la inversión pública es un mecanismo de estabilización económica. El Banco Mundial (Schwartz, Andrés y Dragoiu, 2009) ha calculado que en América Latina el paquete prototipo de proyectos de infraestructura de un monto de 1,000 millones de dólares genera 80,000 puestos de trabajo. Para el caso particular de la construcción de caminos rurales y del mantenimiento de carreteras, este estudio estimo la creación de entre 400,000 y 800,000 empleos correspondientes a 1,000 millones de dólares de gasto. Esto apunta a la creación de un fondo de contingencia para atender la generación de empleos cuando desastres naturales, o crisis de la economía internacional, afectaran la región centroamericana, el cual podría ser alimentado con impuestos al comercio extra regional.

\section{El crecimiento económico requiere ingresos fiscales}

Se debe recalcar que la generación de mayores niveles de capital humano, así como mejorar las instituciones, requiere de sustanciales inversiones $y$, en consecuencia, de la generación de ingresos fiscales adicionales. De acuerdo a los estándares internacionales, el esfuerzo fiscal de El Salvador es muy bajo. Un estudio de Sen Gupta (2008) computó las tasas esperadas de esfuerzo fiscal de una muestra de países latinoamericanos de acuerdo a sus niveles de PIB per cápita, del cual se deduce que el esfuerzo fiscal del país debería haber sido de $20.3 \%$ en 2007 , el cual es alrededor de seis puntos porcentuales arriba de su valor actual, y del mismo orden de magnitud de los valores que han sido recomendados por las instituciones financieras internacionales. De no contar con los recursos fiscales demandados para atender los importantes rubros que determinan el crecimiento y desarrollo económico y social, que radican principalmente en la capacidad institucional y el capital humano, el futuro sería una réplica del estancamiento y deterioro social de las últimas décadas.

Así emerge la urgencia de llevar a cabo una sustancial reforma fiscal que conduzca a lograr un salto significativo en el desarrollo social, y a posicionar el país en una trayectoria sostenida de desarrollo. El elemento crítico de esta reforma debería ser 
la generación de recursos fiscales que permitan eliminar las brechas históricas de atención en materia de salud y educación, ampliando considerablemente la cobertura de los servicios, y el mejoramiento de las instituciones. Es decir, con la reforma fiscal debe ser posible seguir una trayectoria de desarrollo que haga posible alcanzar a Costa Rica y a Panamá en un número dado de años. Es decir, que debe reflejar la aspiración de superar todos los lastres históricos que han restringido el logro de mejores niveles de vida a la sociedad salvadoreña.

La reforma fiscal debería tener un componente de diseminación de los montos adicionales recaudados, los programas a ser atendidos con tales recursos, y la información que estaría disponible cada año al término del ejercicio fiscal sobre la ejecución de los programas, la población atendida y su ubicación, los resultados logrados, etc. Cabe esperar que, al ubicar las finanzas públicas más cerca del ciudadano, éste vaya a tener sentimientos más fuertes de solidaridad y de cohesión social y participación y, a la vez, va a tener una menor oposición a la imposición adicional ${ }^{13}$.

Otro tema de relevancia es la evidencia de que cuando los ciudadanos tienen más información sobre la problemática del país en cuestión, y de las restricciones que enfrentan los ciudadanos que se encuentran en la pobreza, estarán más anuentes a aceptar una reforma fiscal (Fong, 2001).

Un tema adicional en la campaña de "educación fiscal" debe ser insistir en que los pobres no lo son porque no tienen motivación ni aptitud para trabajar, sino que su situación resulta de las grandes restricciones que enfrentan en el marco de la transmisión de la pobreza de generación a generación: los padres que tienen bajos niveles de educación tienen empleos que pagan bajos salarios y en consecuencia no pueden mantener a sus hijos yendo a la escuela. Estos niños entran al mercado laboral a una edad temprana y su limitado nivel de educación no les permite obtener buenos empleos. En consecuencia, sus propios hijos también van a dejar de ir a la escuela y van a vivir en la pobreza como sus padres y abuelos lo hicieron, generando así una trampa de la pobreza ${ }^{14}$.

El punto a recalcar es que superar la trampa de la pobreza requiere inversiones y recursos de gran magnitud. Se debe apuntar que los países que han logrado bajar sustancialmente los niveles de pobreza y alcanzar altas tasas de crecimiento económico tienen altas tasas de esfuerzo fiscal ${ }^{15}$.

Se puede esperar que el aumento de la tasa de crecimiento económico resultante del aumento de la inversión pública contribuya a reducir el crimen, dada la evidencia 
de que existe una relación directa entre el crecimiento económico y la reducción de la violencia presentada por Fanzylber, Lederman y Loayza $(2000)^{16}$. Estos autores calcularon que el aumento de la tasa de crecimiento económico en un punto porcentual está asociado a la reducción de $2.4 \%$ en la tasa de homicidios y de $13,7 \%$ en la tasa de robos. El mayor dinamismo económico conduciría también a reducir la emigración, dada la evidencia de que en América Latina el estancamiento económico incentiva la emigración (Clark, Hatton y Williamson, 2004).

De esa manera, la reforma fiscal debe servir para sustentar la transformación de la economía salvadoreña hacia una de mayor equidad, productividad, empleo y cohesión social ${ }^{17}$.

\section{¿Por qué no crece la economía salvadoreña?}

Un tema que merece atención particular es la explicación del estancamiento económico de la economía salvadoreña. Al tomar en cuenta las altas tasas de crecimiento de las economías de Panamá y Ecuador, se deduce que la dolarización no es la única explicación. Lo que hay que tener en cuenta es que la tasa de inversión en El Salvador es mucho más baja que las de estos dos países, y esto puede explicar su estancamiento. Hay que notar también que la tasa de ahorro nacional de El Salvador es inferior a la de esos países, pues equivale a casi la mitad. De esa manera, al suponer una relación capital-producto de 4, lograr un crecimiento anual del $6 \%$ requeriría que la economía salvadoreña tuviese una tasa de inversión de $24 \%$, la cual sería financiada por el ahorro nacional y el ahorro externo. Dado que el ahorro nacional en el país es de apenas $8 \%$, la tasa de inversión requerida para acelerar el crecimiento demanda una tasa de ahorro externo de $16 \%$, lo cual es virtualmente imposible de lograr sin grandes trastornos económicos, ya que el ahorro externo es igual al déficit en cuenta corriente, el cual no debe superar el $4 \%$. Esto hace ver que la economía se encuentra entrapada en una situación en que no existen los recursos para financiar la inversión. Hay que señalar que en los periodos de dinamismo de la economía salvadoreña

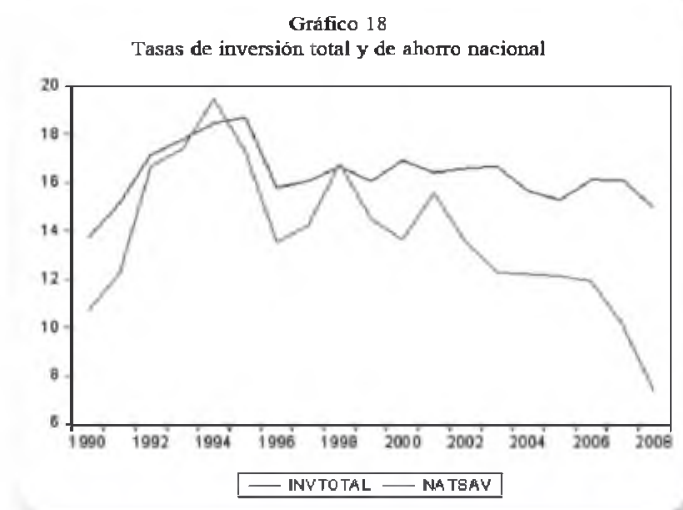


en los años sesenta y setenta, las tasas de ahorro nacional y de inversión eran mucho más altas que las tasas prevalecientes en las últimas décadas.

El gráfico 18 muestra las altas tasas de inversión total, (Invtotal), es decir publica más privada, que la economía salvadoreña experimento en la primera mitad de los años noventa, las que coincidieron con altas tasas de ahorro nacional, Natsav. Se nota en este grafico que el ahorro nacional ha mostrado una caída marcada desde entonces, la que coincide con la caída de la inversión total.

Se debe apuntar que las tasas de ahorro nacional y de inversión de El Salvador, en 2008, fueron las más bajas de Centroamérica, como se aprecia en la tabla 7 :

Tabla 7

Tasas de ahorro y de inversión privada y pública

\begin{tabular}{lrrr}
\hline \multirow{2}{*}{ País } & \multicolumn{3}{c}{ Tasas de $(\%)$ de: } \\
\cline { 2 - 4 } & Ahorro nacional & \multicolumn{2}{c}{ Inversión: } \\
& & Privada & Pública \\
\hline Guatemala & 13.94 & 14.66 & 3.26 \\
El Salvador & 7.35 & 12.82 & 2.13 \\
Honduras & 20.91 & 29.18 & 4.21 \\
Nicaragua & 9.51 & 25.40 & 5.73 \\
Costa Rica & 16.63 & 17.47 & 6.73 \\
\hline
\end{tabular}

Fuente: Banco Mundial, World Development Indicators, varios números

Lo anterior no quiere decir que el consumo privado sea excesivo. Lo que es excesivo es el consumo atendido con bienes importados. Es decir, la baja tasa de ahorro nacional está relacionada con la debilidad de la producción nacional de bienes transables y a su consecuente dependencia en bienes de consumo importados. Si el consumo se atendiera con la producción nacional la economía experimentaría altas tasas de crecimiento económico.
El estancamiento económico de la economía salvadoreña empieza en la segunda mitad de la década de los años noventa, después de la excesiva rebaja de los aranceles a los bienes de consumo importados. Esto ha dado lugar a crecientes déficit en la cuenta comercial, DCOM, (importaciones-exportaciones), que ha ido creciendo continuamente desde $11.66 \%$ en 1997 hasta $22.5 \%$ en 2008. El punto a recalcar es que el déficit comercial incide en la caída 
del ahorro nacional, como se puede observar en el gráfico 19. Este gráfico señala que a medida que el déficit comercial ha ido creciendo el ahorro nacional ha disminuido.

En la actualidad, no existen razones para mantener este grado de apertura irracional que ha traído consecuencias devastadoras para la economía y sociedad salvadoreña. Hay que tomar en cuenta que Panamá y Ecuador tienen aranceles de importación a bienes de consumo más alto que los existentes en El Salvador; este último país los aumentó en el año 2010 como respuesta a la crisis global. En este punto se debe apuntar que, como ha reportado la CEPAL (2009), el arancel efectivo promedio aplicado al sector no agrícola en los países centroamericanos es de $1.2 \%$, inferior a los aplicados en otros esquemas de integración subregional: Comunidad del Caribe: $4.6 \%$; Comunidad Andina, 8.7\%; Mercado Común del Sur: $9.6 \%$. Es irónico que siendo los países centroamericanos los menos desarrollados de la región latinoamericana tienen los aranceles más bajos. Se debe indicar que Rodrik (2001a) ha presentado evidencia para una muestra de países que a mayor protección arancelaria corresponde tasa de crecimiento económico más altas:

"Do lower trade barriers spur greater economic progress? The

Grático 19

Tasa de ahorro nacional (Natsay) y déficit en cuenta comercial (Deom)

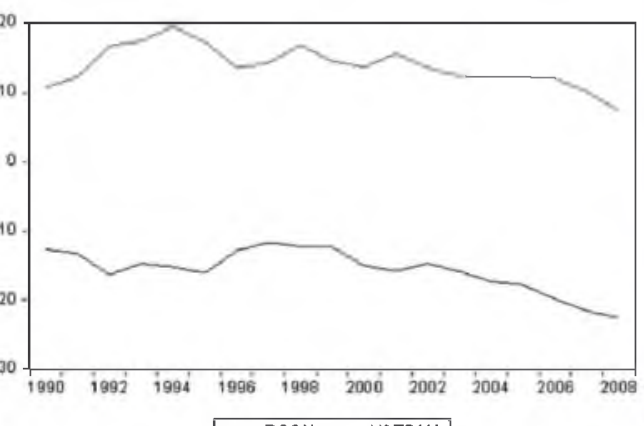

available studies reveal no systematic relationship between a country's average level of tariff and nontariff barriers and its subsequent economic growth rate. If anything, the evidence for the 1990's indicates a positive relationship between import tariffs and economic growth (see chart on opposite page). The only clear pattern is that countries dismantle their trade restrictions as they grow richer. This finding explains why today's rich countries, with few exceptions, embarked on modern economic growth behind protective barriers but now display low trade barriers".

El alto grado de apertura de la economía salvadoreña puede haber sido motivada por las corrientes asociadas al Consenso de Washington, pero hay que señalar que esas corrientes ya han sido repudiadas aun por sus otrora promotores y no cuentan con ningún asidero teórico, ni con evidencia que las sustente a partir de la experiencia de las dos últimas décadas. En efecto, 
discutiendo la ola de reformas que tuvieron lugar en los años noventa en América Latina y otras regiones en vías de desarrollo, Rodrik (2006) ha señalado que:

"The one thing that is generally agreed on about the consequences of these reforms is that things have not quite worked out the way they were intended. Even their most ardent supporters now concede that growth has been below expectations in Latin America... The critics, meanwhile, feel that the disappointing outcomes have vindicated their concerns about the inappropriateness of the standard reform agenda. While the lessons drawn by proponents and skeptics differ, it is fair to say that nobody really believes in the Washington Consensus anymore. The question is not whether the Washington Consensus is dead or alive; it is what will replace it."

Se debe apuntar que el alto déficit comercial requiere, para su financiamiento, además de las remesas, de altos montos de recursos de capital externo; lo que se debe recalcar es la evidencia de que entre mayor es el volumen de recursos externos que un país recibe, menor es su tasa de crecimiento, como ha sido demostrado por Prasad, Rajan y Subramanian (2007). Este resultado inesperado y paradójico apunta otra vez la gran importancia que reside en el ahorro nacional para impulsar el crecimiento, y en corregir la apertura desmedida que agobia a la economía salvadoreña.

Esto señala que para que la economía salvadoreña alcance altas tasas de crecimiento, y así baje el desempleo y subempleo, y la violencia, es necesario corregir su excesiva apertura a las importaciones. Esto implica aumentar los aranceles de importación aplicados a aquellos bienes no comprendidos en los acuerdos de libre comercio (CAFTA y otros). A la par de alentar la inversión en bienes transables, esta medida conduciría a generar recursos fiscales adicionales. Lo anterior podría interpretarse como un regreso a la sustitución de importaciones. Se debe apuntar que fue en el periodo de sustitución de importaciones cuando la economía salvadoreña alcanzó las tasas más altas de crecimiento económico de las últimas décadas y logró, además, avanzar en la industrialización. Recientemente se ha indicado que en términos conceptuales o prácticos, no había nada que le restara valor a la sustitución de importaciones como estrategia de desarrollo (Rodrik, $2001 b)$. Pero no importa el nombre del modelo que se siga, lo que se debe recalcar es el diseño e implementación de políticas con pragmatismo y audacia, teniendo presente que se debe evitar el sacrificio del aparato productivo salvadoreño $y$, a la vez, privilegiar el capital humano y las instituciones, buscando metas que beneficien a todos las y los salvadoreños. 


\section{$\overline{\bar{c}}$ Referencias \\ Abhijit Sen Gupta, "Determinants of Tax Revenue Efforts in Develo- ping Countries", IMF Working Paper, WP/07/184, julio 2007. \\ Alberto Alesina y Robert J. Barro, "Dollarization", American Eco- nomic Review, $\mathrm{N}^{\circ}$ 2, vol. 91, mayo 2001, pp. 381-385.}

Alejandro Castaneda, Pablo Coter y Octavio Gutierrez, "The Impact of Infrastructure in Mexico Manufacturing Growth", Economía mexicana, vol. 9, 2000, pp. 143-164.

Alberto Giovannini, "Savings and the Real Interest Rate in LDCs", Journal of Development Economics, $\mathrm{N}^{\mathrm{o}} 1-2$, vol. 18, 1985, pp. 197-217.

Alain Ize, Eduardo Levy Yeyati y Adrian Azmas, "Financial DoIlarization: The Policy Agenda", IMF, Julio 25, 2006.

Andrew K. Rose, "One Money, One Market: Estimating the Effect of Common Currency on Trade", Economic Policy, vol. 30, abril 2000, pp. 7-33.

Anwar Shah, "Dynamics of Public Infraestructure, Industrial Productivity and Profitability", The Review of Economics and Statistics, vol. LXXIV, 1992, pp. 28-36.
Atish Ghosh, Anne-Marie Gulde, y Holger C. Wolf, "Currency Boards: The Ultimate Fix?" IMF Working Paper WO/98/8, enero de 1998.

Catherine Lynde, "Private Profit and Public Capital", Journal of Macroeconomics, vol. 14, 1992, 125-142.

Christian Broda, "Terms of Trade and Exchange Rate Regimes in Developing Countries", Journal of International Economics, vol. 63, 2004, pp. 31-58.

Chankha Chakraborty y Era DablaNorris, "The Quality of Public Investment", IMF Working Paper No WP/09/154, julio de 2009.

Christine Fong, "Social Preferentes, Self Interest, and the Demand for Redistribution", Journal of Public Economics, vol. 82, 2001, pp. 225-246.

Comision Económica para America Latina y el Caribe, Panorama de la inserción internacional de América Latina y el Caribe, 2008-2009, 2009.

Daniel Kaufmann, Aart Kraay y Máximo Mastruzzi, "Governance Matters VII: Aggregate and Individual Governance Indicators 1996-2007, World Bank Policy Research Working Paper, $N^{\circ} 4654$, junio de 2008. 
Dani, Rodrik, "Why is There so Much Economic Insecurity in Latin America?" Revista de la CEPAL, $N^{\circ}$ 73, abril 2001b, pp. 7-30.

Dani Rodrik, "Goodby Washington Consensus, Hello Washington Confusion?", Journal of Economic Literature, no. 4, vol. 44, diciembre 2006, pp. 973-987.

Dani Rodrik, "Trading in Illusions", Foreign Policy, marzo-abril, 2001a, pp. 55-62.

Dani Rodrik, "Growth after the Crisis", mayo 12, 2009a, inédito.

Dani Rodrik, "The Real Exchange Rate and Economic Growth", Brookings papers on Economic Activity, primavera 2009b.

Dani Rodrik, "Globalization, Social Conflict, and Economic Growth", The World Economy, $N^{\circ}$ 2, vol. 21, marzo 1998, pp. 143-158.

Daron Acemoglu, Simon Johnson, James Robinson y Yunyong Thaicharoen, "Institutional Causes, Macroeconomic Systems: Volatility, Crisis and Growth", Journal of Monetary Economics, vol. 50, pp. 49-123, 2003.

David Hauner y J. Peiris, "Bank Efficiency and Competition in Low Income Countries: The Case of Uganda", IMF Working Paper, WP/05/240, diciembre 2005.
Donahue J. and P. Siegelman, "Allocating Resources Among Prisons and Social Programs in the Battle Against Crime", The Journal of Legal Studies, vol 27, 1998, pp. 1-44.

ECLAC, "The Reactions of Latin America and Caribbean Governments to the International Crisis: An Overview of Policy Measure up to 30 enero 2009", marzo de 2009.

Eduardo Levy-Yeyati, y Federico Sturzenegger, "To Float or to Fix: Evidence on the Impact of Exchange Rate Regimes on Growth", American Economic Review, 2003, pp. 1173-1193.

E. Vegas, y L. Santibañez, The Promise of Early Childhood Development in Latin America and the Caribbean, World Bank, Washington D.C., 2010.

Enrique Aldaz-Carrol y Ricardo Moran, "Escaping the Poverty Trap in Latin America: The Role of Family Factors", Cuadernos de economia, $N^{\circ} 114$, vol. 38, agosto de 2001, pp. 155-190.

Esteban Pérez-Caldentey y Matías Vernengo, "A Tale of two Monetary Reforms: Argentinean Convertibility in Historical Perspective", University of Utah, Department of Economics Working Paper No 2007-01, 2007. 
Eswar Prasad, Raghuram Rajan y Arvind Subramanian, "Foreign Capital and Economic Growth", Brookings Papers on Economic Activity, vol. 1, marzo 2007.

Georgios Karras, "Costs and Benefits of Dollarization: Evidence from North, central, and South America", Journal of Economic Integration, $\mathrm{N}^{\circ} 3$, vol. 17, septiembre de 2002, pp. 502-516.

James E. Anderson y Douglas Marcouiller, "Insecurity and the Pattern of Trade: An Empirical Investigation", The Review of Economics and Statistics, no. 2 , vol. 84, mayo de 2002, pp. 342-352.

Jeffrey P. Cohen y Catherine J. Morrison, "Public Infrastructure Investment, Interstate Spatial Spillovers, and Manufacturing Costs, The Review of Economics and Statistics, vol. 86, 2004, pp. 551-560.

Jordan Z., Schwartz, Luis A. Andres y Georgeta Dragoiu, "Crisis in Latin America: Infrastructure Investment, Employment and the Expectations of Stimulus", Word Bank Working Paper $N^{\circ}$ 5009, julio de 2009.

John Baffes y Anwar Shah, "Productivity of Public Spending, Sectoral Allocation Choices and Economic Growth", Economic Development and Cultural
Change, $N^{\circ} 2$, vol. 46, enero de 1998.

Juan Luis Londoño, y P. Guerrero, Asalto al desarrollo, Banco Interamericano de Desarrollo, 1999.

J. Smith, "The Impact of Childhood Health on Adult Labor Market Outcomes", The Review of Economics and Statistics, $N^{\circ} 3$, vol. 91, 2009.

H. Esfahani y M. Ramirez, "Institutions, Infrastructure, and Economic Growth", Journal of Development Economics, vol. 70, 2003, pp. 443-477.

Kenneth P. Jameson, "Dollarization in Ecuador: A Post-Keynesian Institutionalist Analysis, University of Utah, Department of Economics Working Paper $\mathrm{N}^{\mathrm{o}}$ 2004-5, 2004.

Luis Rene Cáceres, "Inversión y crecimiento económico en Centroamérica", Comercio exterior, №. 6, vol. 47, julio de 1997, pp. 429-436.

Luis René Cáceres, "Exportaciones, inversión y crecimiento económico en Centroamérica", EI trimestre económico, julio de $2007 \mathrm{~b}$

Luis René Cáceres, "Instituciones y Desarrollo Humano", Revista Latinoamericana de desarrollo humano, enero de 2008a. 
Luis René Cáceres, "Instituciones e Integración Economica en Centroamérica", Comercio Exterior, octubre 2008b.

Luis René Cáceres y Patricio Amaya, "Subempleo y pobreza en El Salvador", 2008, sin publicar.

Luis René Cáceres, "Capital humano, instituciones y crecimiento económico en Centroamérica", Comercio exterior, enero de 2009a

Luis René Cáceres, “Economías Centroamericanas: ¿Qué las Hace Crecer?", Comercio Exterior, 2009b.

Luis René Cáceres, “¿Qué variables reducen el desempleo? Evidencia de México y Centroaméri$\mathrm{ca}^{\prime \prime}, 2009 \mathrm{c}$, sin publicar.

L Goldberg y J. Tracy, "Exchange Rates and Local Labor Markets", en The Impact of International Trade on Wages, (Ed.) Feenstra, R. C., University of Chicago Press, Chicago, 2000.

Michael W. Klein, Journal of International Finance and Money, no. 6, vol. 24, octubre 2005, pp. 935-943.

Marco A. Pino-Farah, Gaston Gels y Alejandro Lopez Mejia, "Macroeconomic Implications of $\mathrm{Fi}$ nancial Dollarization: The Case of Uruguay", IMF Occasional Papers, № 263, julio de 2008.

Marigee Bacolod y Priya Ranjan, "Why Children Work, Attend School, or Stay Idle: The Roles of Ability and Household Wealth", Economic Development and Cultural Change, 2008, pp. 827.

Martin Cihak y Richard Podpina, "Bank Behavior in Developing Countries: Evidence from East Africa", IMF Working Paper WP/05/129, junio de 2005.

Mathew Bradbury y Matías Vernengo, "The Limits of Dollarization in Ecuador: Lessons from Argentina", University of Utah, Department of Economics Working Paper No. 2008-12, 2008.

M. Kandil, e I. Mirzaie, "The Effects of Dollar Appreciation on Sectoral Labor Market Adjustments: Theory ad Evidence", The Quarterly Journal of Economics and Finance, vol. 43, 2003, pp. 89-117.

Patrick Iman, "Introducing the Euro as Legal Tender- Benefits and Costs of Eurorization for Cape Verde", IMF Working Paper no. WP/09/146, julio de 2009.

PNUD, Informe Sobre Desarrollo Humano El Salvador 20072008, San Salvador, 2008. 
Richard Esterlin, "Why Isn't the Whole World Developed?", Journal of Economic History, vol. 41, 1981.

Rodney Ramcharan, "Catacysms and Currencies: Does the Exchange Rate Regime Matter for Real Shocks?" IMF Working Paper WP/05/85, abril 2005.

Robert Rennhack, y Masahiro Nozaki, "Financial Dollarization in Latin America", IMF Working Paper WP/06/7, julio 2006.

Shaghil Ahmed, "Sources of Economic Fluctuations in Latin America and Implications for Choice of Exchange Rate Regimes", Journal of Development Economics, vol. 72, 2003, pp. 181-202.

Spence Bocock, "Early Childhood Programs in Other Nations: Goals and Outcomes", The Future of Children, no. 3, Vol. 5, 1995, pp. 94-114.

S. Burges y M. Knetter, "An International Comparison of Employment Adjustment to Exchange Rate Fluctuations", Review of International Economics. No. 1, Vol. 6, 1998, pp.151-164.

Sebastian Edwards y Igal Magendzo, "Dollarization and Economic Performance: What do we rea-
Ily Know?" International Journal of Finance and Economics, $\mathrm{N}^{\circ}$ 4, vol. 84, octubre de 2003, pp. 351-363.

Sebastian Edwards y Igal Magendzo, "Strict Dollarization and Economic Performance: An Empirical Investigation", Journal of Money, Credit and Banking, No1, vol. 38, febrero de 2006, pp. 269-282.

Sebastian Galiani, Daniel Heymann y Mariano Tommasi, "Expectativas frustradas: El ciclo de la convertibilidad" Desarro//O económico, $N^{\circ} 169$, vol 43, abril-junio de 2003, pp. 3-44.

Susan Skipper, "Desarrollo de capital humano y reducción de la pobreza en El Salvador", Comercio exterior, vol. 56, 2006.

T. Beck y H. Hesse, "Why Are Interest Margins so High in Uganda", Journal of Development Economics, No 2, vol. 88, marzo de 2009, pp. 192-204.

World Bank, World Development Indicators, 2004, 2004.

Ximena Clark, Timothy Hatton y Jeffrey Williamson, "What Explains Emigration Out of Latin America?" World Development, No 11, vol. 32, 2004. 


\section{Anexos}

VEnTAJAS Y DESVENTAJAS DE REGÍMENES CAMBIARIOS

a adopción de un régimen cambiario ha sido $\longrightarrow$ un tema de debate en muchos años. Se ha argumentado en defensa de un régimen de tipo de cambio flexible que este dota a la economía de una capacidad de enfrentar disturbios económicos externos a través de la devaluación de la moneda, o sea aumentando el valor de sus exportaciones en moneda local. En favor del régimen de tipo de cambio fijo se ha argumentado que el mismo da lugar a una menor variabilidad del tipo de cambio y así a tasas de inversión y de crecimiento más altas.

Con relación a la evidencia empírica, se debe hacer referencia al estudio de Levy-Yeyati y Sturzenegger (2003) que investigaron el papel del régimen cambiario en una muestra de 183 países con datos del periodo 1974-2000, encontrando que en países desarrollados el régimen cambiario no tiene incidencia sobre el crecimiento económico, mientras que los países en vías de desarrollo que tienen un régimen flexible tienden a crecer a tasas más altas que las de aquellos países con régimen cambiario fijo. También se trae a cuenta el estudio de una muestra de 75 países en vías de de-

sarrollo que concluyo que aquellos con régimen flexible experimentan una menor contracción de la producción cuando experimentan caídas en los términos de intercambio que los países con tipo de cambio fijo, que la diferencia entre las respuestas de ambos regímenes era apreciable solo en el inicio de la reacción de los países y que en el largo plazo ambos régimen cambiarios muestran respuestas similares ante choques de los términos de intercambio (Broda, 2004).

Además, otro estudio analizo las respuestas de una muestra de países latinoamericanos (Argentina, Brasil, Chile, Colombia, México y Venezuela) ante choques externos, concluyendo que no había un caso contundente a favor o en contra de los tipos de cambio fijo o flexible 


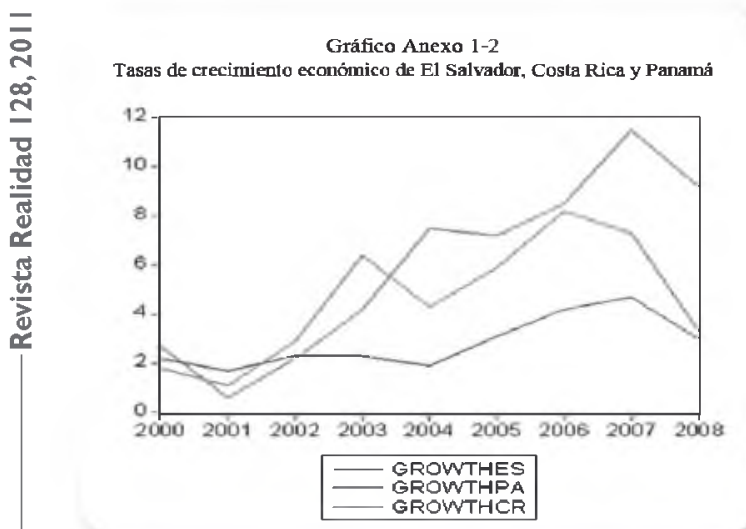

(Ahmed, 2003). Hay evidencia que muestra que países con tipo de cambio fijo tienden a tener tasas de inversión más altas que los países con tipo de cambio flexible (Ramcharan, 2005), lo cual se atribuye a la ausencia de riesgo cambiario. Un punto de vista diferente ha sido argumentado Rodrik (2009b) quien presenta evidencia que altas tasas de crecimiento económico en países en vías de desarrollo están asociadas con monedas depreciadas, y que las aceleraciones de las tasas de crecimiento toman lugar simultáneamente, o son precedidas, de episodios de devaluación. La explicación de este resultado radica en la evidencia de que la depreciación incentiva a las empresas a invertir en el sector de bienes transables, lo que resulta en exportaciones $y$ crecimiento económico acelerado.

Con respecto a estudios de tipo comparativo de economías que han adoptado el dólar como moneda de curso legal, se debe hacer referencia a los estudios de Edwards y
Magendzo (2003; 2006) que analizan grupos de economías totalmente dolarizadas, encontrando que estos países tienden a crecer a tasas más bajas y tienen mayor volatilidad que los países que tienen sus propias monedas. ${ }^{18}$

Sobre estudios de costos y beneficios de dolarización, se debe mencionar el de Estudio de Alesina and Barro (2001) sobre las condiciones que deben ser cumplidas por los países que están considerando la dolarización; la exanimación de los países que cumplían estas condiciones indico que un grupo de países Africanos podrían adoptar el Euro; algunos países Asiáticos se beneficiarían de adoptar el Euro; y un pequeño grupo de países latinoamericanos podrían beneficiarse de adoptar el dólar de los EEUU. Los beneficios considerados en el estudio eran la eliminación de la variabilidad del tipo de cambio y los ahorros en la reducción de costos de transacción. Otra propuesta de costos y beneficios de la dolarización la ha presentado Karras $(2002)^{19}$.

También debe hacerse referencia a estudios ${ }^{20}$ que han encontrado que cuando países tienen una moneda común el monto de su comercio reciproco tiende a aumentar significativamente, en un multiplicador de dos o tres. Sin embargo, Klein (2005) ha reportado evidencia 
empírica de que el comercio entre países totalmente dolarizados y los EEUU no ha mostrado ningún dinamismo especial, siendo de la misma magnitud que el de los países con tipo de cambio fijo.

Ubicando esta evidencia en el contexto de las economías centroamericanas se puede esperar que sus tasas de inversión tuvieran relación con su régimen cambiario; específicamente los países con tipos de cambio fijo tendrían tasas de inversión más altas, lo que daría lugar a tasas de crecimiento más dinámicas ${ }^{21}$. Sin embargo, se debe notar que una economía dolarizada como Panamá, tiene tasas de inversión y de crecimiento económico más altas que las de El Salvador y los otros países centroamericanos. Así mismo, Costa Rica, que tiene un tipo de cambio flexible, muestra tasas de inversión y de crecimiento más altas que las de El Salvador (Gráficos Anexo1, 1 y 2). Esto indica que hay otros factores además del régimen cambiario que afectan en las tasas de inversión y de crecimiento económico de una manera crucial.

\section{Determinación del diferencial de tasa de interés}

N o existe una teoría única sobre la determinación del diferencial de tasas activa y pasiva; un punto de vista común es que este diferencial depende del balance de los bancos, particularmente de las relaciones de liquidez y solvencia, y también de la penetración de estos en el mercado, y en las condiciones macroeconómicas como las tasas de inflación y de crecimiento económico. ${ }^{22}$ Varias ecuaciones fueron estimadas para identificar las variables que determinan el diferencial de tasas de interés, y los resultados se muestran en la tabla 2-1 de este anexo. La ecuación (1) muestra el diferencial como función del aumento mensual del Ivae, la tasa Repo, el aumento del cuasi-dinero (M2(-1) - M1 (-1), y del aumento mensual del índice de precios al consumidor. Los resulta- dos indican que el único coeficiente que es significativo es el del Ivae; este coeficiente es negativo, indicando que cuando aumenta el índice de actividad económica, lo que estimula la demanda de crédito, el diferencial cae, lo que se puede interpretar como consecuencia de la competencia entre los bancos. El coeficiente del cuasi-dinero es negativo, lo que denota que la mayor liquidez resultante de aumentos en los depósitos en los bancos, conduce a mejorar sus balances y resultados, y así a reducir los spreads. $\mathrm{O}$, alternativamente, puede indicar que aumentar la tasa de depósito, dejando la tasa activa sin cambio, lo cual reduce el diferencial, resulta en aumentar los montos de depósitos atraídos por la tasa de depósito más alta. Sin embargo, este coeficiente es significativo solo al nivel 
de $10 \%$. Nótese que el coeficiente del aumento del Ipc es positivo, lo que denota que cuando la inflación aumenta, los bancos protegen sus ganancias aumentando el diferencial. Sin embargo, este coeficiente es significativo solo al nivel de $10 \%$. El coeficiente de la tasa Repo es negativo, contrario a lo esperado, pero no es significativo.

La ecuación (2) introduce una variable adicional, la diferencia entre la tasa activa en dólares de Guatemala, RLENDG, y la tasa Repo de El Salvador ${ }^{23}$. Esta nueva variable muestra un coeficiente que es significativo, indicando que a medida de que la tasa activa de Guatemala aumenta en relación con la tasa Repo, la banca salvadoreña se vuelve más atractiva como fuente de financiamiento para agentes económicos de Guatemala. Se puede notar que el coeficiente de esta variables es muy significativa, y que los coeficientes de las otras variables se vuelven más significativos que en la ecuación (1), y que el coeficiente de Repo tiene un signo positivo como era de esperar.

La ecuación (3) introduce la diferencia entre la tasa activa en dólares de Honduras, RLENDH, y la tasa Repo de El Salvador. Esta variable muestra un coeficiente muy significativo, y todas las otras variables, excepto el aumento del índice de precios al consumidor, tienen coeficientes que son significativos al nivel de cinco por ciento.

Cuando la diferencia entre la tasa activa de Nicaragua en dólares, RLENDN, y la tasa Repo se incluye en la ecuación (4) se puede ver que su coeficiente es significativo, pero los niveles de significancia de las otras variables disminuyen, excepto el aumento del Ivae, pero mantienen el signo esperado.

La ecuación (5) muestra que la diferencia entre la tasa active en dólares de Costa Rica, RLENDCR, y la tasa repo no es significativa, ni tampoco lo son los coeficientes de las otras variables. Lo que indica que no hay efecto de Costa Rica hacia el diferencial de El Salvador.

Nótese que el grado de significancia estadística de los coeficientes de la diferencia de tasas activa y Repo disminuye a medida de que aumenta la distancia desde El Salvador ${ }^{24}$.

Estos resultados indican que la disponibilidad de dólares en El Salvador motiva a agentes económicos de los otros países centroamericanos a buscar financiamiento en la plaza salvadoreña cuando sus tasas activas son altas en relación con la tasa Repo de El Salvador. Esto señala el potencial que existe para que El Salvador se vuelva un centro financiero internacional. 
Tabla anexo 2-1.

Determinantes del spread

\begin{tabular}{|c|c|c|c|c|c|}
\hline & (1) & $(2)$ & (3) & (4) & (5) \\
\hline \multirow[t]{2}{*}{ Constante } & 3,4784 & 0.2044 & 1.8258 & 1.9527 & 3.3892 \\
\hline & -24.97 & -0.35 & -4.27 & -4.6 & -3.68 \\
\hline \multirow[t]{2}{*}{ D(IVAE) } & -0.0062 & -0.0049 & -0.0057 & -0.0066 & -0.0062 \\
\hline & -1.9 & -1.8 & -1.91 & -2.15 & -1.86 \\
\hline \multirow[t]{2}{*}{ REPO } & -0.0626 & 0.3573 & 0.136 & 0.117 & -0.0562 \\
\hline & -1.46 & -4.41 & -2.15 & -1.89 & -0.72 \\
\hline \multirow[t]{2}{*}{$\mathrm{D}(\mathrm{M} 2(-1)-\mathrm{M} 1(-1)-0.0012$} & -0.0014 & -0.0013 & -0.0011 & -0.0012 & \\
\hline & -1.77 & -2.35 & -1.98 & -1.76 & -1.76 \\
\hline \multirow[t]{2}{*}{$\mathrm{D}(\mathrm{IPC})$} & 0.054 & 0.05136 & 0.0643 & 0.053 & 0.0541 \\
\hline & -1.29 & -1.48 & -1.68 & -1.39 & -1.28 \\
\hline (RLENDG-REPO) & & $\begin{array}{r}0.4618 \\
-5.77\end{array}$ & & & \\
\hline (RLENDH-REPO) & & & $\begin{array}{r}0.158 \\
-4.05 \\
\end{array}$ & & \\
\hline (RLENDN-REPO) & & & & $\begin{array}{r}0.1154 \\
-3.77\end{array}$ & \\
\hline (RLENCR-REPO) & & & & & $\begin{array}{r}0.0102 \\
-0.09\end{array}$ \\
\hline R cuadrado & 0.15 & 0.43 & 0.31 & 0.29 & 0.16 \\
\hline D.W. & 0.66 & 1 & 0.84 & 0.79 & 0.66 \\
\hline
\end{tabular}




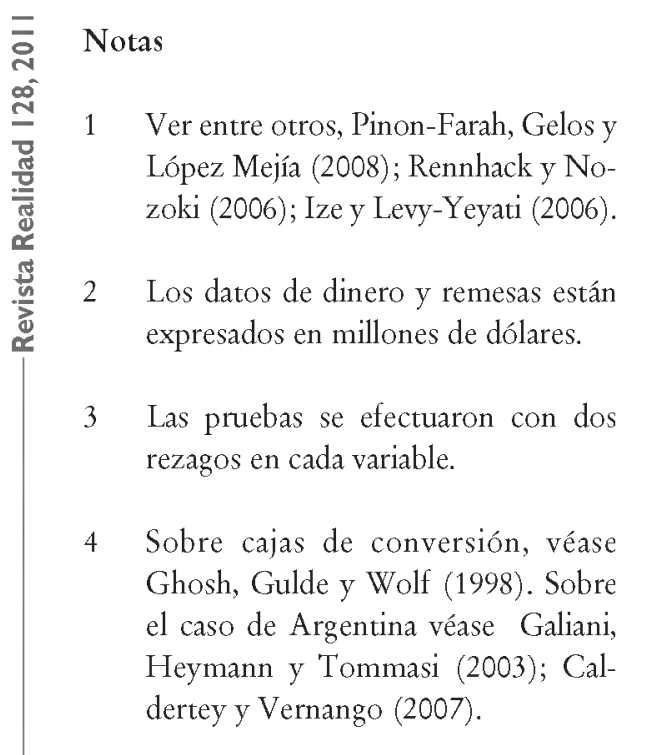

5 Véase reseñas de Acemoglu et. al. (2003).

6 Reseñas de la literatura se encuentran en Caceres (2008b; 2009a)

7 Aldaz-Carrol y Moran (2002), Bocock (1995), Bacolod y Ranjan (2008).

8 Smith (2009), Vegas y Santibanez (2010).

9 Sobre el papel de las exportaciones en el crecimiento económico de El Salvador véase Caceres (2007b)

10 Los datos son del periodo 1990-2003 y los de las exportaciones están expresados en millones de dólares de 1990.

11 Sobre el caso de Mexico, vease Castaneda, Cotler y Gutiérez (2000) y Shah (1992).
12 Sobre los rendimientos de la inversión pública en infraestructura véase entre otros, Lynde (1992), Esfahani y Ramírez (2003), Castañeda, Coter y Gutiérrez (2000), y Cohen y Paul (2004).

13 Se debe hacer referencia al estudio de Cáceres $(2009$ c) que mostróque si la carga impositiva fuese aumentada en 3 puntos porcentuales y si todos los recursos fuesen destinados a la inversión pública, la economía adquiriría a una tasa adicional de crecimiento económico anual de 0.77 puntos porcentuales. La mayor tasa de crecimiento generaría una economía de mayor tamaño que aquella en ausencia del aumento de los impuestos, de manera que en 2017 la economía que resulta con el aumento de impuestos es de mayor dimensión que la economía sin mayor carga impositiva, aú después de deducir los impuestos pagados.

14 Sobre la trampa de pobreza en El Salvador, véase Caceres y Amaya (2008).

15 Los porcentajes de ingresos fiscales a PIB en algunos países de América Latina fueron en 2008: Brasil 24.8, Panamá 18.0, Chile 27.0, Costa Rica 15.6. En algunos países se han introducido novedosos mecanismos, como el impuesto a las transacciones financieras en Republica Dominicana, Brasil y Costa Rica, y el sistema de tasas diferenciadas del Iva en Colombia, con tasas que van de 5 a 40 por ciento. 
16 Estos autores concluyeron que: "By increasing the availability of job opportunities and raising wages in the legal vis-a-vis the criminal labor market, economic growth has a crime reducing effect" (página 246).

17 Hay que notar la reforma fiscal recientemente aprobada en Costa Rica, según informa La Prensa Grafica del 17 de enero de 2011, que entre otros elementos comprende el aumento del impuesto a las ventas de 13 a $15 \%$, así como la eliminación de bienes exentos de impuestos.

18 Un análisis de la dolarización en Ecuador desde el punto de vista post-keynesiano se encuentra en Jameson (2004). Véase también sobre Ecuador a Bradbury y Vernengo (2008).

19 Un análisis de los costos y beneficios de la adopción del Euro como moneda en Cabo Verde se encuentra en Iman (2009).
20 Ver Rose (2000).

21 Existe evidencia de que en los países centroamericanos las tasas de inversión son los principales determinantes del crecimiento económico, vease Caceres (1997, 2009b).

22 Ver, entre otros, Beck y Hesse (2009), Hauner y Peiris (2005), Cihak y Podpina (2005).

23 Datos de las tasas de interés activas en dólares de los otros países centroamericanos se obtuvieron de la Secretaría Ejecutiva del Consejo Monetario Centroamericano, www. secmca.org

24 Los estadísticos " $T$ " de los coeficientes (Relend - Repo) correspondientes a Guatemala, Honduras, Nicaragua y Costa Rica son, respectivamente, $5.77,4.5,3.77$ y 0.09 . 